\title{
Titanium carboxylate complexes stability constants estimated by four graphical methods
}

\author{
Abdalazeem. A. Omar *, Elmugdad. A. Ali \\ Department of chemistry, faculty of science, Sudan University of Science and Technology, Sudan \\ *Corresponding authorE-mail: abdeenomar78@hotmail.com
}

Copyright $\odot 2014$ Abdalazeem. A. Omar; Elmugdad. A. Ali. This is an open access article distributed under the Creative Commons Attribution License, which permits unrestricted use, distribution, and reproduction in any medium, provided the original work is properly cited.

\begin{abstract}
This investigation represents a comparative study of four graphical methods for overall stability constant estimation techniques, included point wise calculation method, half integral method, linear plot method and least squares method, in purpose to select the most sensitive and accurate method, the paper aiming also to determine the parameters that affect the precision of these methods under investigation.

Metal-ligand stability constant and stoichiometries of above systems are to be determined by Calvin and Bjerrum $\mathrm{pH}$ metric titration technique as adopted by Irving and Rossotti. For this purpose titanium has been chosen as a central metal ion. Titanium element from first transition series of d-block and has four valence bond electrons $3 \mathrm{~d} 24 \mathrm{~s} 2$.

Acetic acid, oxalic acid and oxalacetic acid have been selected as ligands. The obtained results seem in a good agreement with each other, with some restriction on least squares method for accumulation of error in Kn-1. These suggestions have been introduced in conclusion for treatment of data by least squares method and Henderson's Hasselbalch's equation and other modifications have been done.
\end{abstract}

Keywords: Oxalacetic Acid, Titanium Caboxylates, Graphical Methods, Mat-Lab Program.

\section{Introduction}

Many method of great diversity are now being used for determination of step-wise stability constants. However the most widely used and the most accurate and reliable method for determination of stability constant are based on potentiometric measurement of hydrogen ion concentration. The magnitude of the observed $\mathrm{pH}$ change may be employed to determine the stability constant of the metal complex by Bjerrum's is used by Calvin and Wilson's method [1]. Some of these methods used to calculate the stability constants, have been reviewed recently by Sullivan and Hindman [2]. Fundamental assumption that the complex formation is stepwise process, if this true then the ratios of various stability constants could in theory is predicted by statistical considerations [3].

The study of metal-ligand complexes in a solution would be of interest which throw a light on the mode of storage and transport of metal ion in biological kingdom with a view to understand the bio-inorganic chemistry of the metal ions [4]. The proton-ligand and metal-ligand stability constants are strongly affected by ionic strength of the medium [5]. Dissociation of organic acid and their interactions with metal ions (complex formation) may be extremely sensitive to ionic strength of the medium. If charges in the reacting species are opposite then there is a decrease in the reaction rate with increasing ionic strength, whereas if the charges are identical, an increase in the reaction rate will occur, and if one of the reactants is charge less the reaction rate will not be affected by ionic strength of the medium. This could be related to the concentration of electrolytes and indicates how effective the charge on a particular ion is shielded or stabilized by other ions in an electrolyte [6].

\section{Material and methods}

\subsection{Preparation of stock solution}


All materials used in this investigation were chemically pure brand and hence were used without further purification. The solutions used throughout the experiments were prepared in doubly distilled water.

All measurements were carried out with $\mathrm{pH}$-meter Denver instrument ultra-basic $\mathrm{pH} / \mathrm{mV}$ meter with combine electrode at $29 \pm 1^{\circ} \mathrm{C}$, the sensitivity of the $\mathrm{pH}$-meter is 0.01 units, the instrument could read $\mathrm{pH}$ in range 0.00 to $14.00 \mathrm{in}$ step 0.01 . The $\mathrm{pH}$-meter was switched on before half an hour for warm up before starting titration. The instrument was calibrated before each set of titration using two buffer solutions of $\mathrm{pH} 4$ and 10. The electrode was washed with distilled water and dried with tissue paper. The readings were recorded only when the instrument registered a steady value for at least one minute.

\subsection{Procedure}

Three set of solutions total volume of each (V) $200 \mathrm{~cm}^{3}$ were prepared for titration against free carbonate sodium hydroxide solution. The change in $\mathrm{pH}$ of solution with each addition of alkali was recorded for each of the following mixtures to calculate values of formation functions $\mathrm{n}_{\mathrm{A}}, \mathrm{n}^{-}, \mathrm{pL}$ (where $\mathrm{n}_{\mathrm{A}}$ average number of proton associated with ligand, $\mathrm{n}^{-}$the average number of ligand attached to metal ion and $\mathrm{pL}$ is the free ligand exponential function) [1].

A/ Free acid. (A)

$\mathrm{C} /$ Free acid + ligand. $(\mathrm{A}+\mathrm{L})$

$\mathrm{D} /$ Free acid + ligand + metal ion. $(\mathrm{A}+\mathrm{L}+\mathrm{M})$

All solutions were completed with double distilled water to $200 \mathrm{~cm}^{3}$, after adding $1 \mathrm{~cm}^{3}$ of potassium nitrate solution to maintain ionic strength constant, the titrations were carried out in $250 \mathrm{~cm}^{3}$ beaker with magnet bar inside for stirring the solution.

On plotting the observed $\mathrm{pH}$ against the volume of alkali added, different trends have got, Acid curve (A), a ligand curve $(\mathrm{A}+\mathrm{L})$ lies below the acid curve indicating the dissociation of ligand in the reaction medium, and metal complex curve $(\mathrm{A}+\mathrm{L}+\mathrm{M})$ lies below the ligand titration curve indicating the complex formation.

A matlab program have been created for plotting the graph for volume of alkali solution versus $\mathrm{pH}$ of each three set of solutions (acid, acid+ligand and acid+ligand+metal ion) and secondly to find the volume of alkali required for bring each of three set of solutions to the same $\mathrm{pH}$. Calvin and Wilson have demonstrated $\mathrm{pH}$ measurement made during titration with alkali solution of ligand in presence and absence of metal ion could be employed to calculate the formation functions $\mathrm{n}_{\mathrm{A}}, \mathrm{n}^{-}$and $\mathrm{pL}$.

The values of formation functions were calculated by excel program on personal computer according to the expressions 1,2 and 3 [7].

${ }^{-}{ }_{A}=y-\frac{\left(V_{1}-V_{2}\right)\left(N^{0}+E^{0}\right)}{\left(V^{0}+V_{1}\right) T_{C L}^{0}}$

Where $\mathrm{y}=$ the number of dissociable protons, $\mathrm{N}^{0}$ is the concentration of the alkali, $\mathrm{E}^{0}$ is the concentration of the free acid, $T_{C L}^{0}$ is the total ligand concentration, $\mathrm{V}^{0}$ is the total volume of titration solution, $\mathrm{V}_{1}$ and $\mathrm{V}_{2}$ the volume of alkali add to acid and acid + ligand respectively bring each of them to same $\mathrm{pH}$ value.

The average number of the ligand to metal or metal-ligand formation number at various $\mathrm{pH}$ values determined according to Irving and Rossotti by the following equation:

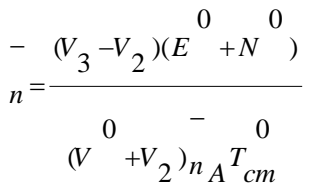

Where $\mathrm{V}_{2}$ and $\mathrm{V}_{3}$ the volume of alkali required to mixture of (acid + ligand) and (acid+ligand +metal) to bring them to same $\mathrm{pH}$ value, $\mathrm{T}_{\mathrm{cm}}$ is the total concentration of the metal and other significances as in equation (1).

A free ligand exponent function (PL) was calculated using equation (3) [8].

$$
P L=\log _{10}\left\{\frac{1+\beta_{n}^{H} \frac{1}{[\operatorname{anti} \log \mathrm{pH}]^{\mathrm{n}}}}{\mathrm{T}_{\mathrm{CL}}^{0}-{ }_{n T}^{0}} \times \frac{\left(V^{0}+V_{3}\right)}{V^{0}}\right\}
$$

Where $\mathrm{V}_{3}=$ volume of alkali required to bring the solution of the complex to same $\mathrm{pH}$ in titration curve.

\section{Result and discussion}

\section{1 proton-ligand stability constant}




\subsubsection{Poinwise calculation method}

The calculation of free ligand exponent function $(\mathrm{pL})$ of metal complexes requires a prior knowledge of the practical proton-ligand stability constant [9] (equation 3). Using excel program on personal computer the values of $n_{A}$ at various $\mathrm{pH}$ reading $(\mathrm{B})$ were calculated from the acid and ligand titration curve. For acetic acid the ligand titration curve is well separated from the free acid titration curve at $\mathrm{pH} 2.5$,oxalic acid at $\mathrm{pH} 2.3$ and oxalacetic acid at $\mathrm{pH}=2.3$ indicating the dissociation of the ligand in the titration medium [10].

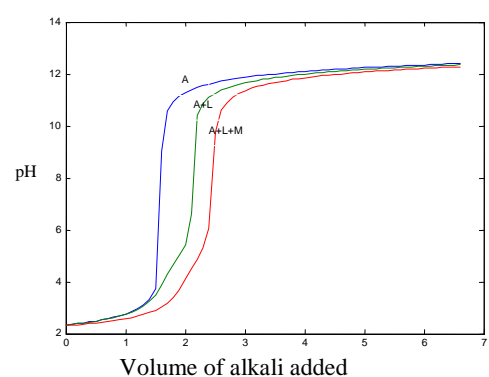

Fig. 1: Formation Curves of Three Set of Solutions. A-Free Acid (Hno3), L-Ligand (Acetic Acid), M-Metal Ion (Titanium Chloride)

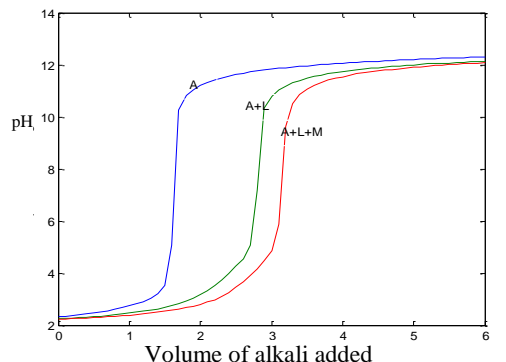

Fig. 2: Titration Curves of Three Set of Solutions. A-Free Acid (Hno3), L-Ligand (Oxalic Acid), M-Metal Ion (Titanium Chloride) (Titanium Chloride).

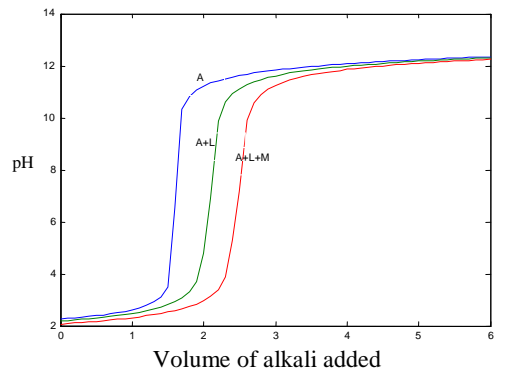

Fig. 3: Titration Curves of Three Set of Solutions. A-Free Acid(Hno3), L-Ligand (Oxalacetic Acid), M-Metal Ion (Titanium Chloride)

The formation number for the ligands extends $0<\mathrm{n}_{\mathrm{A}}<1,0<\mathrm{n}_{\mathrm{A}}<1.6$ and between 1.4267 and 0.6656 in $\mathrm{n}_{\mathrm{A}}$ scale for acetic, oxalic and oxalacetic acid respectively. According to point-wise calculation method the dissociation constants were calculated for acetic acid, oxalic acid and oxalacetic acid on tables below. The values of $\mathrm{pK}_{\mathrm{a} 1}$ and $\mathrm{pKa}_{2}$ were taken as an average on range of 0.2 to 0.8 and 1.2 to 1.8 on $\mathrm{n}_{\mathrm{A}}$ scale respectively [11].

Table 1: Proton-Ligand Stability Constant $\left(\mathrm{Pk}_{\mathrm{a}}\right)$ of Acetic Acid. Average $\mathrm{Pk}_{\mathrm{a}}=4.669$

\begin{tabular}{|c|c|c|c|c|c|}
\hline B & V1 & V2 & $\mathrm{n}_{\mathrm{A}}$ & $\log \left(\frac{n_{A}}{\left(1-n_{A}\right)}\right)$ & $\mathrm{pKa}$ \\
\hline 3.6 & 1.4674 & 1.5308 & 0.8894 & 0.9055 & 4.5055 \\
\hline 3.7 & 1.4907 & 1.5564 & 0.8854 & 0.8881 & 4.5881 \\
\hline 3.8 & 1.5011 & 1.5821 & 0.8588 & 0.7839 & 4.5839 \\
\hline 3.9 & 1.5030 & 1.6071 & 0.8185 & 0.6541 & 4.5541 \\
\hline 4.0 & 1.5049 & 1.6310 & 0.7801 & 0.5500 & 4.5500 \\
\hline 4.1 & 1.5068 & 1.6548 & 0.7420 & 0.4587 & 4.5587 \\
\hline 4.2 & 1.5087 & 1.6786 & 0.7038 & 0.3758 & 4.5758 \\
\hline 4.3 & 1.5106 & 1.7028 & 0.6649 & 0.2976 & 4.5976 \\
\hline 4.4 & 1.5125 & 1.7306 & 0.6197 & 0.2121 & 4.6121 \\
\hline 4.5 & 1.5143 & 1.7583 & 0.5746 & 0.1306 & 4.6306 \\
\hline 4.6 & 1.5162 & 1.7861 & 0.5294 & 0.0512 & 4.6512 \\
\hline 4.7 & 1.5181 & 1.8143 & 0.4836 & -0.0285 & 4.6715 \\
\hline 4.8 & 1.5200 & 1.8429 & 0.4371 & -0.1099 & 4.6901 \\
\hline 4.9 & 1.5219 & 1.8714 & 0.3907 & -0.1930 & 4.7070 \\
\hline 5.0 & 1.5238 & 1.9000 & 0.3441 & -0.2801 & 4.7199 \\
\hline 5.1 & 1.5257 & 1.9233 & 0.3068 & -0.3539 & 4.7461 \\
\hline 5.2 & 1.5275 & 1.9465 & 0.2695 & -0.4330 & 4.7670 \\
\hline 5.3 & 1.5294 & 1.9698 & 0.2322 & -0.5193 & 4.7807 \\
\hline
\end{tabular}

Table 2: Determination of Log Pka1 of Oxalic Acid (Average Logka1=1.4645)

\begin{tabular}{ccc}
\hline $\mathrm{pH}$ & $\mathrm{n}_{\mathrm{A}}$ & \\
\hline 2.1 & 1.9735 & 0.5342 \\
2.2 & 1.9473 & 0.9457 \\
2.3 & 1.1958 & 3.9135 \\
2.4 & 1.1884 & 3.0343 \\
2.5 & 1.1675 & 3.1964 \\
2.6 & 1.1552 & 3.5022 \\
2.7 & 1.1362 & 3.6627 \\
2.8 & 1.1206 & 3.8488 \\
2.9 & 1.1011 & 4.0214 \\
3 & 1.0869 & 4.2548 \\
3.1 & 1.0654 & 4.5315 \\
3.3 & 1.0445 & 5.0970 \\
- & 1.0157 & - \\
\end{tabular}


Table 3: Determination of $\log \mathrm{Pk}_{\mathrm{a} 2}$ of Oxalic Acid (Average $\mathrm{Pka}_{2}=4.5869$ )

\begin{tabular}{ccc}
\hline $\mathrm{pH}$ & $\mathrm{n}_{\mathrm{A}}$ & $\log \mathrm{Ka}_{2}$ \\
\hline 3.7 & 0.8638 & 4.5022 \\
3.8 & 0.8169 & 4.4494 \\
3.9 & 0.7653 & 4.4134 \\
4.0 & 0.7178 & 4.4056 \\
4.1 & 0.6605 & 4.3890 \\
4.2 & 0.6136 & 4.4008 \\
4.3 & 0.5676 & 4.4182 \\
4.4 & 0.5295 & 4.4514 \\
4.5 & 0.4886 & 4.4801 \\
4.6 & 0.4500 & 4.5129 \\
4.7 & 0.4159 & 4.5525 \\
4.8 & 0.3876 & 4.6014 \\
4.9 & 0.3631 & 4.6560 \\
5.0 & 0.3421 & 4.7159 \\
5.1 & 0.3221 & 4.7768 \\
5.2 & 0.3114 & 4.8553 \\
5.3 & 0.3006 & 4.9333 \\
5.4 & 0.2899 & 5.0108 \\
\hline
\end{tabular}

Table 4: Determination of Log Pka 1 of Oxalacetic Acid (Average $\left.\mathrm{Pka}_{2}=2.8967\right)$

\begin{tabular}{|c|c|c|c|c|c|}
\hline B & V1 & V2 & $\mathrm{n}_{\mathrm{A}}$ & $\log \left(\frac{n_{A}}{\left(1-n_{A}\right)}\right)$ & $\mathrm{pK}_{\mathrm{a} 2}$ \\
\hline 2.4 & 0.3000 & 0.625 & 1.4267 & -0.1283 & 2.2717 \\
\hline 2.5 & 0.5750 & 0.875 & 1.4715 & -0.0495 & 2.4505 \\
\hline 2.6 & 0.7667 & 1.0667 & 1.4720 & -0.0487 & 2.5514 \\
\hline 2.7 & 0.9286 & 1.2167 & 1.4934 & -0.0115 & 2.6885 \\
\hline 2.8 & 1.0556 & 1.3556 & 1.4728 & -0.0473 & 2.7527 \\
\hline 2.9 & 1.1500 & 1.4900 & 1.4028 & -0.1711 & 2.7289 \\
\hline 3.0 & 1.2267 & 1.5500 & 1.4323 & -0.1183 & 2.8817 \\
\hline 3.1 & 1.2933 & 1.6267 & 1.4148 & -0.1495 & 2.9504 \\
\hline 3.2 & 1.3321 & 1.6933 & 1.3661 & -0.2384 & 2.9616 \\
\hline 3.3 & 1.3679 & 1.7391 & 1.3487 & -0.2714 & 3.0286 \\
\hline 3.4 & 1.4013 & 1.7826 & 1.3311 & -0.3055 & 3.0945 \\
\hline 3.5 & 1.4143 & 1.8177 & 1.2923 & -0.3840 & 3.1160 \\
\hline 3.6 & 1.4273 & 1.8471 & 1.2636 & -0.4462 & 3.1539 \\
\hline 3.7 & 1.4403 & 1.8765 & 1.2349 & -0.5129 & 3.1871 \\
\hline 3.8 & 1.4532 & 1.9035 & 1.2102 & -0.5748 & 3.2251 \\
\hline 3.9 & 1.4662 & 1.9211 & 1.2022 & -0.5961 & 3.3039 \\
\hline
\end{tabular}

Table 5: Determination of Log $\mathrm{Pka}_{2}$ of Oxalacetic Acid (Average $\left.\mathrm{Pka}_{1}=10.6056\right)$

\begin{tabular}{|c|c|c|c|c|c|}
\hline B & V1 & $\mathrm{V} 2$ & $\mathrm{n}_{\mathrm{A}}$ & $\log \left(\frac{\left(n_{A}-1\right)}{\left(2-n_{A}\right)}\right)$ & $\mathrm{pK}_{\mathrm{a} 1}$ \\
\hline 8 & 1.5658 & 2.2401 & 0.8180 & 0.6527 & 8.6527 \\
\hline 8.3 & 1.5709 & 2.2511 & 0.8077 & 0.6232 & 8.9232 \\
\hline 8.6 & 1.576 & 2.262 & 0.7975 & 0.5954 & 9.1954 \\
\hline 8.9 & 1.5812 & 2.273 & 0.7874 & 0.5686 & 9.4686 \\
\hline 9.2 & 1.5863 & 2.2839 & 0.7773 & 0.5428 & 9.7428 \\
\hline 9.5 & 1.5914 & 2.2949 & 0.7670 & 0.5173 & 10.017 \\
\hline 9.8 & 1.5966 & 2.3191 & 0.7340 & 0.4401 & 10.240 \\
\hline 10.1 & 1.6137 & 2.3548 & 0.7012 & 0.3705 & 10.470 \\
\hline 10.4 & 1.6548 & 2.3905 & 0.7109 & 0.3908 & 10.791 \\
\hline 10.7 & 1.6959 & 2.4595 & 0.6623 & 0.2926 & 10.993 \\
\hline 11.0 & 1.79 & 2.5653 & 0.6425 & 0.2545 & 11.255 \\
\hline 11.3 & 1.9751 & 2.7501 & 0.6442 & 0.2579 & 11.558 \\
\hline 11.6 & 2.3335 & 3.1144 & 0.6363 & 0.2430 & 11.843 \\
\hline 11.9 & 3.0337 & 3.8005 & 0.6656 & 0.2989 & 12.199 \\
\hline
\end{tabular}

\subsection{Metal ligand stability constant: (Ti-acetate, Ti-oxalate and Ti-oxalacete)}

\subsubsection{Poinwise calculation method}

Metal titration curve showed a displacement with respect to the ligand titration curve along the volume axis for acetic acid at $\mathrm{pH}$ 2.4, oxalic acid at $\mathrm{pH} 2.4$, and oxalacetic acid at $\mathrm{pH} 2.3$, figure (1), figure (2) and figure (3) indicating the affinity of ligand with metal ions which release a protons and produced the volume difference $\left(\mathrm{V}_{3}-\mathrm{V}_{2}\right)$ [12], which utilized to calculate the values $\mathrm{n}^{-}$and $\mathrm{pL}$ and further to evaluate the metal-ligand stability constants. Calculation of $\mathrm{n}^{-1} \mathrm{~s}$ values, showed that titanium-acetic acid system forms two complexes $\mathrm{ML}_{2}$ and $\mathrm{ML}_{3}$. 
Table 6: Point Wise Calculation Method Logk (Average $\operatorname{Logk}_{2}=5.4153$ (Titanium Acetate)

\begin{tabular}{|c|c|c|c|c|c|}
\hline B & V2 & V3 & $\mathrm{n}^{-}$ & $\mathrm{pL}$ & $\log K_{2}$ \\
\hline 2.5 & 0.5250 & 0.8000 & 1.4582 & 5.4532 & 5.3805 \\
\hline 2.6 & 0.7600 & 1.0400 & 1.5421 & 5.3786 & 5.4518 \\
\hline 2.7 & 0.9286 & 1.2286 & 1.6193 & 5.3032 & 5.5145 \\
\hline 2.8 & 1.0625 & 1.3714 & 1.6933 & 5.2283 & 5.5825 \\
\hline 2.9 & 1.1700 & 1.4889 & 1.7455 & 5.1473 & 5.6141 \\
\hline 3.0 & 1.2539 & 1.5818 & 1.8029 & 5.0691 & 5.6790 \\
\hline 3.1 & 1.3235 & 1.6533 & 1.8227 & 4.9781 & 5.6446 \\
\hline 3.2 & 1.3824 & 1.7150 & 1.8623 & 4.8951 & 5.6919 \\
\hline 3.3 & 1.4280 & 1.7650 & 1.8711 & 4.8012 & 5.6310 \\
\hline
\end{tabular}

Table 7: Pointwise Calculation Method Log Average Logk $_{3}=4.2640$ (Titanium Acetate)

\begin{tabular}{|c|c|c|c|c|c|}
\hline $\mathrm{B}$ & V2 & V3 & $\mathrm{n}^{-}$ & $\mathrm{pL}$ & $\mathrm{K}_{3}$ \\
\hline 3.6 & 1.5308 & 1.8742 & 2.0192 & 4.5745 & 2.8667 \\
\hline 3.7 & 1.5564 & 1.9047 & 2.0570 & 4.4978 & 3.2793 \\
\hline 3.8 & 1.5821 & 1.9279 & 2.1054 & 4.4283 & 3.4995 \\
\hline 3.9 & 1.6071 & 1.9512 & 2.1979 & 4.3851 & 3.7772 \\
\hline 4.0 & 1.6310 & 1.9744 & 2.3010 & 4.3564 & 3.9904 \\
\hline 4.1 & 1.6548 & 1.9977 & 2.4156 & 4.3483 & 4.2002 \\
\hline 4.2 & 1.6786 & 2.0225 & 2.5537 & 4.3826 & 4.4763 \\
\hline 4.3 & 1.7028 & 2.0475 & 2.7090 & 4.4889 & 4.8758 \\
\hline 4.4 & 1.7306 & 2.0725 & 2.8824 & 4.8070 & 5.6823 \\
\hline
\end{tabular}

Titanium-oxalic acid system forms two chelates $\mathrm{ML}_{2}$ and $\mathrm{ML}_{3}$.

Table 8: $\operatorname{Logk}_{2}$ by Point Wise Method (Average $\operatorname{Logk}_{2}=5.081$; Ti-Oxalate)

\begin{tabular}{|c|c|c|c|c|c|}
\hline $\mathrm{B}$ & $\mathrm{V} 2$ & V3 & $\mathrm{n}^{-}$ & $\mathrm{pL}$ & $\log K_{2}$ \\
\hline 2.5 & 1.1000 & 1.4000 & 1.5170 & 4.9825 & 5.0120 \\
\hline 2.6 & 1.3400 & 1.6500 & 1.5909 & 4.9024 & 5.0620 \\
\hline 2.7 & 1.5167 & 1.8500 & 1.7489 & 4.8530 & 5.3277 \\
\hline 2.8 & 1.6625 & 2.0000 & 1.7778 & 4.7632 & 5.3072 \\
\hline 2.9 & 1.7778 & 2.1200 & 1.8361 & 4.6856 & 5.3933 \\
\hline
\end{tabular}

Table 9: Determination of $\operatorname{Logk}_{3}$ by Point-Wise Method (Average Logk ${ }_{3}=4.1907$; Ti-Oxalate)

\begin{tabular}{|c|c|c|c|c|c|}
\hline $\mathrm{B}$ & $\mathrm{V} 2$ & V3 & $\mathrm{n}^{-}$ & $\mathrm{pL}$ & $\log K_{3}$ \\
\hline 3.3 & 2.0875 & 2.4211 & 2.0570 & 4.3957 & 3.1772 \\
\hline 3.4 & 2.1400 & 2.4737 & 2.1460 & 4.3478 & 3.5807 \\
\hline 3.5 & 2.1900 & 2.5227 & 2.2236 & 4.3006 & 3.7601 \\
\hline 3.6 & 2.2348 & 2.5682 & 2.3911 & 4.3202 & 4.1281 \\
\hline 3.7 & 2.2783 & 2.6125 & 2.6296 & 4.4535 & 4.6840 \\
\hline 3.8 & 2.3200 & 2.6542 & 2.8983 & 4.9357 & 5.8816 \\
\hline
\end{tabular}

Titanium-oxalacetic acid system forms only one chelate $\mathrm{ML}_{2}$.

Table 10: Logk $_{2}$ of Titanium Oxalacelate by Point Wise Method. Average Logk $=8.6384$

\begin{tabular}{|c|c|c|c|c|c|}
\hline $\mathrm{B}$ & $\mathrm{V} 2$ & V3 & $\mathrm{n}^{-}$ & $\mathrm{pL}$ & $\log K_{2}$ \\
\hline 2.7 & 1.3714 & 1.7445 & 1.2653 & 11.1601 & 10.7178 \\
\hline 2.9 & 1.575 & 1.9500 & 1.3132 & 10.8617 & 10.5207 \\
\hline 3.1 & 1.7091 & 2.0824 & 1.3438 & 10.5817 & 10.3010 \\
\hline 3.3 & 1.800 & 2.1708 & 1.3937 & 10.3286 & 10.1411 \\
\hline 3.5 & 1.8513 & 2.2250 & 1.4059 & 10.0841 & 9.91856 \\
\hline 3.7 & 1.9009 & 2.2635 & 1.4418 & 9.86084 & 9.75929 \\
\hline 3.9 & 1.9191 & 2.3007 & 1.5409 & 9.66712 & 9.73838 \\
\hline 4.1 & 1.9373 & 2.3152 & 1.5503 & 9.45522 & 9.54288 \\
\hline 4.3 & 1.9555 & 2.3297 & 1.5600 & 9.24861 & 9.35326 \\
\hline 4.5 & 1.9736 & 2.3442 & 1.5701 & 9.04559 & 9.16824 \\
\hline 4.7 & 1.9918 & 2.3587 & 1.5805 & 8.84485 & 8.98585 \\
\hline 4.9 & 2.0052 & 2.3732 & 1.6010 & 8.64871 & 8.82653 \\
\hline 5.1 & 2.0147 & 2.3877 & 1.6297 & 8.45618 & 8.68677 \\
\hline 5.3 & 2.0242 & 2.4016 & 1.6558 & 8.26357 & 8.54358 \\
\hline 5.5 & 2.0337 & 2.4120 & 1.6670 & 8.06659 & 8.36824 \\
\hline 5.7 & 2.0431 & 2.4225 & 1.6789 & 7.87011 & 8.19525 \\
\hline 5.9 & 2.0526 & 2.4330 & 1.6907 & 7.67379 & 8.02268 \\
\hline 6.1 & 2.0621 & 2.4435 & 1.7026 & 7.47763 & 7.85100 \\
\hline 6.3 & 2.0716 & 2.4539 & 1.7142 & 7.28145 & 7.67915 \\
\hline 6.5 & 2.081 & 2.4644 & 1.7265 & 7.08561 & 7.50986 \\
\hline 6.7 & 2.0905 & 2.4749 & 1.7408 & 6.89054 & 7.34666 \\
\hline 6.9 & 2.100 & 2.4853 & 1.7559 & 6.69584 & 7.18684 \\
\hline 7.1 & 2.1067 & 2.4958 & 1.7767 & 6.50323 & 7.04456 \\
\hline 7.3 & 2.1135 & 2.5044 & 1.7889 & 6.30773 & 6.88027 \\
\hline 7.6 & 2.1236 & 2.5154 & 1.7987 & 6.01161 & 6.61002 \\
\hline
\end{tabular}


What should be mentioned, during titration course of oxalacetic acid a precipitation occurred at low values of $\mathrm{pH}$, and then disappear at high values of $\mathrm{pH}$ which indicates Henderson's or Hasselbalch's equation will be eligible for determination of stability constant of this chelate. [3]

Every two consecutive complex species having appreciable difference $(\geq 1.8)$ i.e. $\log K_{2}$ and $\log K_{3}$ values, this difference indicate chelates or complexes were occurred in stepwise process, and not simultaneously.[13] that is not the case in this investigation. Titanium-oxalacetic acid system forms one chelate $\mathrm{ML}_{2}$ if it compared with titanium oxalic acid which formed two chelates $\mathrm{ML}_{2}$ and $\mathrm{ML}_{3}$, one can say this may refer to the longer chain of oxalacetic acid relative to oxalic acid chain, that might leave no room for complex $\mathrm{ML}_{2}$ (titanium oxalacetate) to accommodate extra ligand. These values obtained by this method were further verified by half integral method.

\subsubsection{Half integral method: (Ti-acetates, Ti-oxalates and Ti-oxalacetate)}

For estimation of metal-ligand stability constants, a formation curve was constructed by plotting $\mathrm{n}^{-}$against $\mathrm{pL}$. The values of $\mathrm{pL}$ at which $\mathrm{n}^{-}=1.5$ and $\mathrm{n}^{-}=2.5$ corresponds to values of $\log \mathrm{K}_{2}$ and $\log \mathrm{K}_{3}$ respectively. [11]

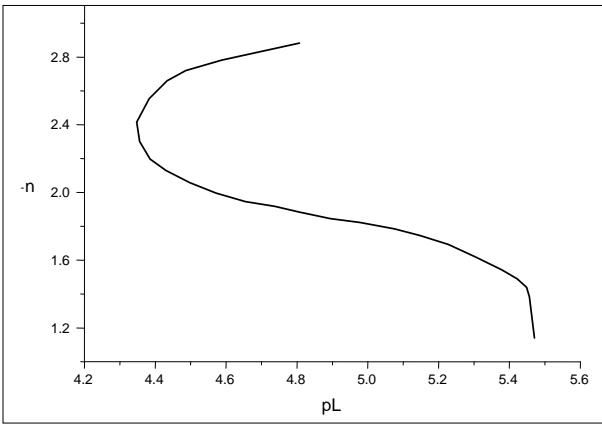

Fig. 4: Metal-Ligand Formation Curve (Titanium Acetate). Logk2=5.412, Logk3=4.352 -Half Integral Method

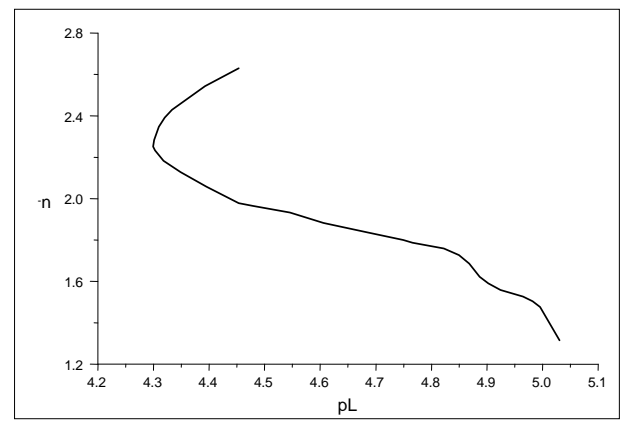

Fig. 5: Metal-Ligand Formation Curve (Titanium Oxalate). Logk2= 4.986 and Logk3= 4.376 -Half Integral Method.



Fig. 6: Metal-Ligand Formation Curve (Titanium Oxalacetate). Logk $2=9.76$ Half Integral Method

The values were in a good agreement with those obtained by point-wise method. These values were further verified by linear plot method.

\subsubsection{Linear plot method: (Ti-acetates, Ti-oxalates and Ti-oxalacetate)}

In this method $\log \left(\mathrm{n}^{-}-1\right) /\left(2-\mathrm{n}^{-}\right), \log \left(\mathrm{n}^{-}-2\right) /\left(3-\mathrm{n}^{-}\right)$, etc. were plotted versus corresponding $\mathrm{pL}$ for determination of $\log \mathrm{K}_{2}$, $\log \mathrm{K}_{3} \ldots$ etc [14]. The values of stability constants obtained for titanium acetate complexes were estimated from figures (7) and (8).

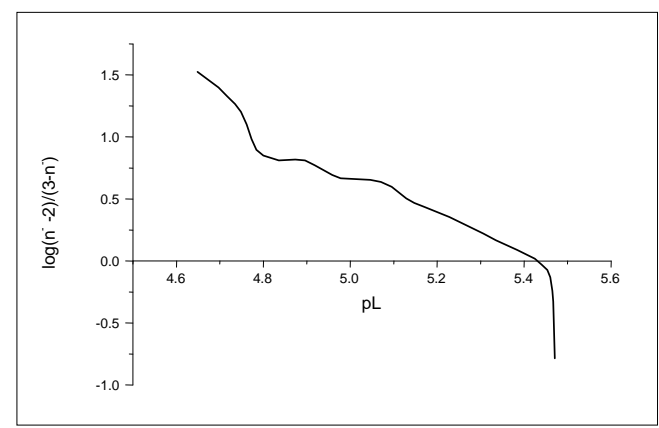

Fig. 7: Linear Plot Method Logk2 =5.427 (Titanium Acetate)



Fig. 8: Linear Plot Method Logk3=4.36 (Titanium Acetate) 
The values of $\log \mathrm{K}_{2}$ and $\log \mathrm{K}_{3}$ for titanium oxalate chelates were estimated from figures (9) and (10).

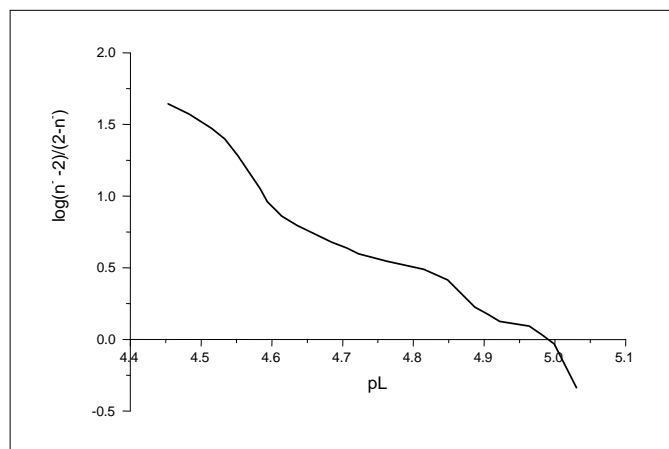

Fig. 9: Linear Plot Method $\operatorname{Logk}_{2}=4.991$ (Titanium Oxalate)

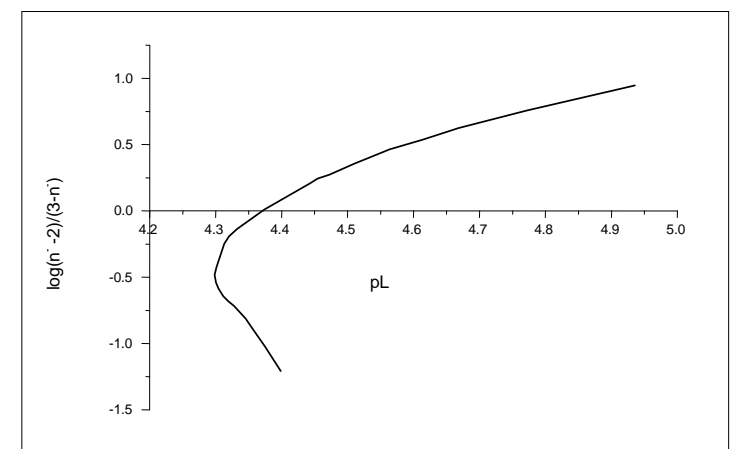

Fig. 10: Linear Plot Method $\operatorname{Logk}_{3}=4.375$ (Titanium Oxalate)

The stability constant of titanium oxalacetate chelate was found from figure (11).

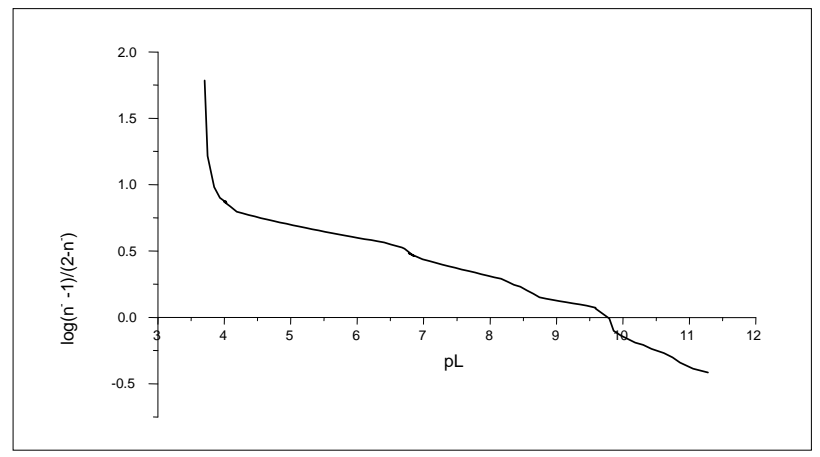

Fig. 11: Linear Plot Method $\operatorname{Logk}_{2}=9.821$. ( Titanium Oxalacetat

The values obtained were in a good concordance with the other values of two previous methods, point wise calculation method and half integral one.

The neutral salt $\mathrm{KNO}_{3}$ that used to maintain the ionic strength constant has very slight complexing tendency, and the competition between nitrate and the ligands under study are of minor importance [14]. These values were further verified by least squares method but the value of titanium oxalacetate was verified by equation familiar as Henderson's or Hasselbalch's equation.

\subsubsection{Least squares method: (Ti-acetate and Ti-oxalate)}

For estimation of $\mathrm{K}_{2}$ and $\mathrm{K}_{3}$ for titanium acetate and titanium oxalate by this method, linear equation of Rossotti and Rossotti as expressed below with some modification to meet our purpose has been employed:

$\frac{\left(n^{-}-1\right)}{\left(2-n^{-}\right)[L]}=\frac{\left(3-n^{-}\right)[L]}{\left(2-n^{-}\right)} \times K_{2} K_{3}-K_{3}$

If the values of $n^{-}$and [L] were known, plotting of $\left(n^{-}-1\right) /\left(2-n^{-}\right)[L] v / s\left(3-n^{-}\right)[L] /\left(2-n^{-}\right)$, give straight line equation with $\mathrm{y}$-intercept $=\mathrm{K}_{3}$ and slope $=\mathrm{K}_{2} \mathrm{~K}_{3}$.

The other form of equation (4) can be obtaining on division by $\mathrm{K}_{2} \mathrm{~K}_{3}$ and this give nearly the same values $\mathrm{x}$-intercept $=1 / \mathrm{K}_{2}$ and slope $1 / \mathrm{K}_{2} \mathrm{~K}_{3}$ which useful check on the consistency of the data.

$$
\frac{\left(n^{-}-1\right)}{\left(2-n^{-}\right)[L]} \times \frac{1}{K_{2} K_{3}}+\frac{1}{K_{2}}=\frac{\left(3-n^{-}\right)[L]}{\left(2-n^{-}\right)}
$$




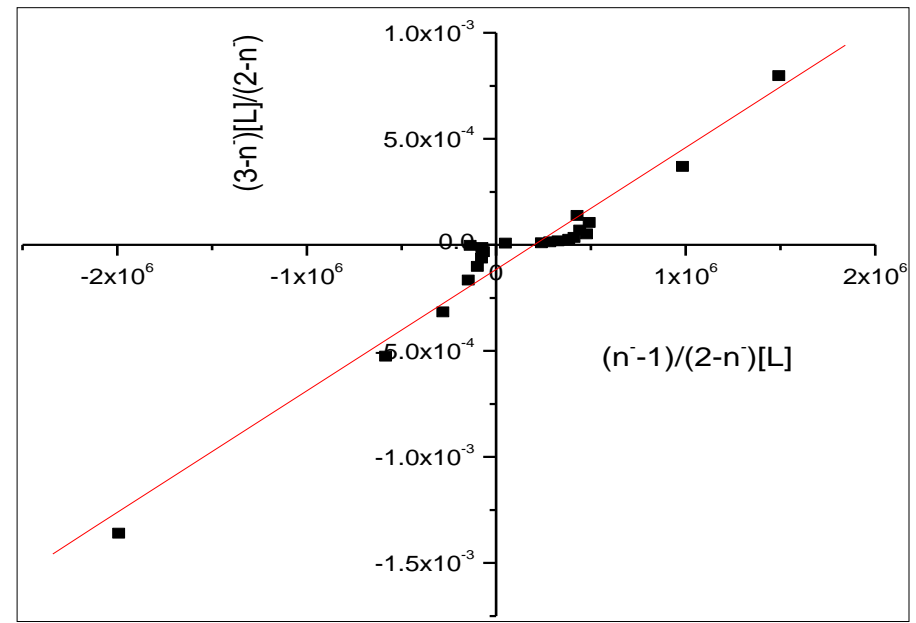

Fig. 12: Least Squares Method $\operatorname{Logk}_{2}=5.2368 \& \operatorname{Logk}_{3}=4.3010$. (Titanium Acetate)

\begin{tabular}{llll} 
& \multicolumn{3}{c}{ Linear Regression for: $\mathrm{Y}=\mathrm{A}+\mathrm{B} * \mathrm{X}$} \\
\hline Parameter & Value & Error & $\mathrm{R}^{2}$ \\
\hline Intercept & -0.0001 & 0.00002 & 0.97962 \\
Slope & $5.7975 \mathrm{E}-10$ & $2.80147 \mathrm{E}-11$ & 0 \\
\hline
\end{tabular}

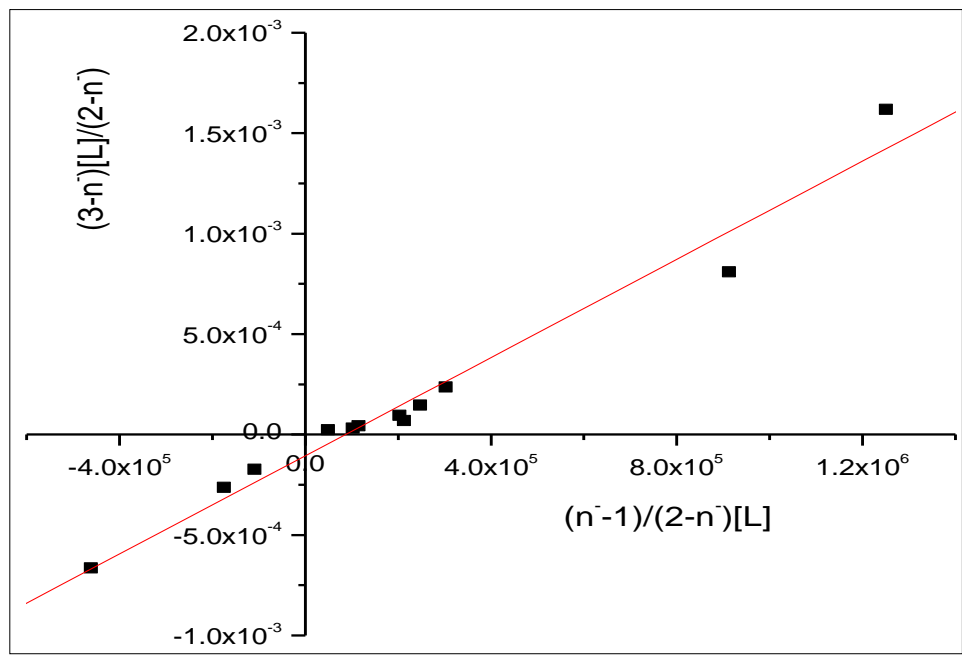

Fig. 13: Least Squares Method, $\operatorname{Logk}_{2}=4.913 \& \operatorname{Logk}_{3}=4.3010$. (Titanium Oxalate)

\begin{tabular}{|c|c|c|c|}
\hline Parameter & Value & Error & $\mathrm{R}^{2}$ \\
\hline Intercept & -0.0001 & 0.00003 & 0.9854 \\
\hline Slope & $1.22219 \mathrm{E}-9$ & $6.67725 \mathrm{E}-11$ & \\
\hline
\end{tabular}

Any errors in the values of $\beta_{1} \ldots \beta_{\mathrm{t}-1}$ will accumulate in the value of $\beta_{\mathrm{t}}$. (Where $\left.\mathrm{t}=\mathrm{N}-1\right)$ [2].

\subsubsection{Henderson's or hasselbalch's equation: (Ti-oxalacetate system)}

This equation is used when only one complex or chelate is formed.

$\log \left(\frac{n^{-}}{\left(1-n^{-}\right)}\right)=\log \beta_{1}+\log [L]$

The equation is familiar as Henderson's or Hasselbalch's equation, and has been widely used for determination of the dissociation constants of monobasic acids. It is also useful for determining constants from $\mathrm{n}^{-}$and [L] data over a very limited range e.g. if very strong or very weak complexes are formed, or if precipitation occurs at low value of $n^{-}$which is the case in this investigation. [3] 
Table 11: $\operatorname{Logk}_{2}$ by Henderson's or Hasselbalch's Equation. Average $\operatorname{Logk}_{2}=8.6384$ (Titanium Oxalacetate)

\begin{tabular}{|c|c|c|c|c|c|c|c|c|c|}
\hline $\mathrm{n}^{-}$ & {$[\mathrm{L}]$} & $\log \left(\frac{\left(n^{-}-1\right)}{\left(2-n_{A}\right)}\right)$ & $\log ([\mathrm{L}])$ & $\log \mathrm{K}_{2}$ & $\mathrm{n}^{-}$ & [L] & $\log \left(\frac{\left(n^{-}-1\right)}{\left(2-n_{A}\right)}\right.$ & $\log ([\mathrm{L}])$ & $\log \mathrm{K}_{2}$ \\
\hline 1.2653 & $6.92 \mathrm{E}-12$ & -0.4423 & -11.1601 & 10.7178 & 1.6439 & $4.36 \mathrm{E}-09$ & 0.2573 & -8.3602 & 8.6175 \\
\hline 1.2712 & $9.92 \mathrm{E}-12$ & -0.4293 & -11.0035 & 10.5741 & 1.6558 & 5.45E-09 & 0.2800 & -8.2636 & 8.5436 \\
\hline 1.3132 & $1.38 \mathrm{E}-11$ & -0.3409 & -10.8617 & 10.5207 & 1.6616 & $6.84 \mathrm{E}-09$ & 0.2912 & -8.1651 & 8.4563 \\
\hline 1.3412 & $1.90 \mathrm{E}-11$ & -0.2858 & -10.7222 & 10.4364 & 1.6670 & 8.58E-09 & 0.3017 & -8.0666 & 8.3682 \\
\hline 1.3438 & $2.62 \mathrm{E}-11$ & -0.2807 & -10.5817 & 10.3010 & 1.6733 & $1.08 \mathrm{E}-08$ & 0.3140 & -7.9684 & 8.2824 \\
\hline 1.3754 & $3.51 \mathrm{E}-11$ & -0.2211 & -10.4543 & 10.2332 & 1.6789 & $1.35 \mathrm{E}-08$ & 0.3251 & -7.8701 & 8.1952 \\
\hline 1.3937 & $4.69 \mathrm{E}-11$ & -0.1875 & -10.3286 & 10.1411 & 1.6850 & $1.69 \mathrm{E}-08$ & 0.3374 & -7.7720 & 8.1094 \\
\hline 1.4275 & $6.14 \mathrm{E}-11$ & -0.1268 & -10.2119 & 10.0851 & 1.6907 & $2.12 \mathrm{E}-08$ & 0.3489 & -7.6738 & 8.0227 \\
\hline 1.4057 & $8.24 \mathrm{E}-11$ & -0.1655 & -10.0841 & 9.9186 & 1.6964 & $2.66 \mathrm{E}-08$ & 0.3605 & -7.5756 & 7.9361 \\
\hline 1.4214 & $1.07 \mathrm{E}-10$ & -0.1377 & -9.9702 & 9.8325 & 1.7026 & $3.33 \mathrm{E}-08$ & 0.3734 & -7.4776 & 7.8510 \\
\hline 1.4418 & $1.38 \mathrm{E}-10$ & -0.1016 & -9.8608 & 9.7593 & 1.7083 & 4.17E-08 & 0.3854 & -7.3795 & 7.7649 \\
\hline 1.4934 & $1.73 \mathrm{E}-10$ & -0.0115 & -9.7632 & 9.7517 & 1.7142 & $5.23 \mathrm{E}-08$ & 0.3977 & -7.2815 & 7.6791 \\
\hline 1.5409 & $2.15 \mathrm{E}-10$ & 0.0713 & -9.6671 & 9.7384 & 1.7204 & $6.55 \mathrm{E}-08$ & 0.4111 & -7.1835 & 7.5946 \\
\hline 1.5459 & $2.75 \mathrm{E}-10$ & 0.0799 & -9.5605 & 9.6404 & 1.7265 & $8.21 \mathrm{E}-08$ & 0.4242 & -7.0856 & 7.5099 \\
\hline 1.5503 & $3.51 \mathrm{E}-10$ & 0.0877 & -9.4552 & 9.5429 & 1.7327 & $1.03 \mathrm{E}-07$ & 0.4378 & -6.9877 & 7.4256 \\
\hline 1.5554 & $4.45 \mathrm{E}-10$ & 0.0966 & -9.3515 & 9.4481 & 1.7408 & $1.29 \mathrm{E}-07$ & 0.4561 & -6.8905 & 7.3467 \\
\hline 1.5600 & $5.64 \mathrm{E}-10$ & 0.1046 & -9.2486 & 9.3533 & 1.7481 & $1.61 \mathrm{E}-07$ & 0.4728 & -6.7931 & 7.2659 \\
\hline 1.5654 & 7.13E-10 & 0.1143 & -9.1469 & 9.2612 & 1.7559 & 2.01E-07 & 0.4910 & -6.6958 & 7.1868 \\
\hline 1.5701 & $9.00 \mathrm{E}-10$ & 0.1226 & -9.0456 & 9.1682 & 1.7664 & $2.51 \mathrm{E}-07$ & 0.5161 & -6.5996 & 7.1156 \\
\hline 1.5754 & $1.13 \mathrm{E}-09$ & 0.1319 & -8.9450 & 9.0769 & 1.7767 & $3.14 \mathrm{E}-07$ & 0.5413 & -6.5032 & 7.0446 \\
\hline 1.5805 & $1.43 \mathrm{E}-09$ & 0.1410 & -8.8449 & 8.9859 & 1.7857 & $3.92 \mathrm{E}-07$ & 0.5641 & -6.4065 & 6.9706 \\
\hline 1.5862 & $1.80 \mathrm{E}-09$ & 0.1513 & -8.7453 & 8.8966 & 1.7889 & $4.92 \mathrm{E}-07$ & 0.5725 & -6.3077 & 6.8803 \\
\hline 1.6010 & $2.25 \mathrm{E}-09$ & 0.1778 & -8.6487 & 8.8265 & 1.7926 & $6.18 \mathrm{E}-07$ & 0.5823 & -6.2092 & 6.7914 \\
\hline 1.6149 & $2.80 \mathrm{E}-09$ & 0.2032 & -8.5522 & 8.7553 & 1.7954 & 7.76E-07 & 0.5897 & -6.1103 & 6.7000 \\
\hline 1.6297 & $3.50 \mathrm{E}-09$ & 0.2306 & -8.4562 & 8.68687 & 1.7987 & $9.74 \mathrm{E}-07$ & 0.5984 & -6.0116 & 6.6100 \\
\hline
\end{tabular}

The higher the values of stability constants showed that the ligands under investigation are strong chelating agents [15].

\section{Conclusion}

The four graphical methods give nearly the same values for each titanium caboxylate system. The values of stability constants estimated by point-wise method, half integral method and linear plot method were in a good agreement specially half integral and linear plot method, but the results obtained by least squares method were slightly different specially in $\mathrm{K}_{\mathrm{n}-1}$, where the error accumulated, this method has many restriction which can be found in literature. Reader no doubt by now may ask the question "what method can I use, and which should give me the most accurate value". Each of four methods employed above seen satisfactorily to give the answer, with difference $\leq 0.2$. For a system where $\mathrm{N} \leq 3$, half integral method and linear plot one, give nearly unique answer for $\mathrm{K}_{\mathrm{n}}$ values, whereas common fault is that, as $n^{-}$increase, $K_{n}$ becomes more prone to error. Differences in magnitude between $K_{n}$ values are due solely to errors inherent in the experimental data or non-rigorous data treatment, that is, least squares fit to curves with correlated errors.

In least squares method the slope and intercept obtained by adding trend line and display equation on chart is less accurate than that obtained by LINEST in excel program, or that obtain from origin program (view- result log or fit linear). In each case if the intercept and slope were divided by 2 as this found by extremely trail, the $K_{n}$ value agreed with other values obtained by different methods and this minimize the error accumulated in $\mathrm{K}_{\mathrm{n}-1}$, this which has been done throughout this investigation. In Henderson's equation the stability constant should be taken as an average for values of e.g $\log \mathrm{K}_{2}$ in the range 1.2-1.8, in this way the value of stability constant meet values obtained by other methods. For all results $\mathrm{K}_{2}>\mathrm{K}_{3}$ this order implies no specific electronic or steric phenomenon.

\section{Acknowledgement}

I would like to thank that who assisted me throughout this work and stand behind me always my sincere friend Dr. Abdallah Aissa, for his valuable observation and his large knowledge of ways of publication, during his reading to this work. Thanks also extend to Professor Elmugdad A. Ali for his helpful suggestion and continuous encouragement and direction. Thanks also to department of chemistry, faculty of Science, University of northern border for using equipments.

\section{References}

[1] E-book: Gurdeep, Raj, 1986.Advance inorganic chemistry- II , first ed. Satyendra Rastogi "Mitra" p.67.Book code : 277-12 Ninth thoroughly revised 2004Twelfth ed. 2010

[2] F. J. C. Rossotti, H. S. Rossotti. 1955. Graphical methods for determining equilibrium constants.1- system of mononuclear complexes. Ac. chem. scandinavica. 9, 1166-1176. 
[3] F.R.,'Hartley, C. Burgess, R.M. Alcock.1980. Solution equilibria, first ed. John Wiley and sons, New York.

[4] A.K. Maldhure, S.S. Agarkar, R.K. Taywade, R.S. Wankhade. (2012). Metal-ligand stability constants of $\mathrm{Co}$ (II), $\mathrm{Ni}$ (II ) and $\mathrm{Cu}$ (II) metal ion complexes with N-(5-methyl-2-hydroxyacetophenone)-N-(2-hydroxyacetophenone).J. Chem. pharm. Res. 4, (8), 3865-3868.

[5] Sumer D. Thakur, Devidas, T. Mahajan, Kamlesh P. Munot, Rajesh, D. Deshmukh. 2011a. Studies in influence of ionic strength of medium on complex equilibria in substituted hydroxyl-1-3-propandiones with $\mathrm{Cr}$ (III) and La (III) metal ions pH-metrically. Der pharma chem. 3, (6), 165-173.

[6] Santosh, D. Deosarkar, Ashok, B. Kalambe, Vijay, J. Thakare, Shivraj, G. Wanale, Vinay, V. Hiwase. 2011. Potentiometric studies of electrolyte effects on complex equilibria of some substituted 5-(2-hydroxy phenyl) Pyrazoles. Der pharma chemic. 3, (6), 75-83.

[7] Minaxi, Maru, M.K. Shah. 2011. Synthesis and physic-chemical studies of some divalent transition metal complexes of 2-(2'-chloro-6'substituted quinoliny)-1H-benzo[d]imidazole ligands. J. Chem. Res. 2, (2), 14-27.

[8] Ashwini, kumar, Ashok, Kumar, Malik. 2007. Potentiometric studies on the complexes of Cu ( II ), Cd ( II ), Co ( II ), $\mathrm{Ni}$ ( II ), $\mathrm{Pb}$ ( II ), and Zn (II) with Gabapentin. Euras. J. analy. Chem. 2, (2), 1-6.

[9] Avinash, Ramteke, Marutil, Narwade. 2013. Studies of stability constants of complexes of chlorosubstituted pyrazoles and pyrazoline with $\mathrm{Cu}$ ( II ), $\mathrm{Ni}$ ( II ), Co ( II ) and Nd ( II ) metal ions in 70\% dioxane- water mixture at 0.1 ionic strength. Arch. of appl. Sc. 5, (1), 231-237.

[10] Abdulbaset, A. Zaid, Mazahar, Farooqui, D.M. Janrao. 2012. Study of stability constant of biological active molecules (drug) using potentiometric titration technique. J. of chem. boils. phys. Sc. 2, (1), 67-81.

[11] Sayyed, Hussain, Abdul Rahim, Mazahar, Farooqui. 2011. Studies of binary complexes of metal ions with mandelic acid by potentiometry. Chem. J. 02, (6), 206-209.

[12] G. V., Mane, L.P. shinde, D.G. Kolhatkar. 2011. pH-metric study of proton-ligand and metal-ligand stability constant complexes of transition and inner transition metal ions with some organic acids.Inter. J. Chemistry. 3, (2), 181-187.

[13] Sumer, D. Thakur, Devidas, T. Mahajan, Kamlesh, P. Munot, Rajesh D. Deshmukh, Mangesh, S. Tihile. 2011b. Metal-ligand stability constants of Th (III), Sm (III), Nd (III) and Pr (III) metal ion complexes with two mercapto-4-substituted phenyl-6-substituted phenyl prymidines at $0.1 \mathrm{M}$ ionic strength $\mathrm{pH}$-metrically. Der pharma chem. 3, (6), 382-389.

[14] Anjani, Kumar, Mishra, Prem, Mohan, Mishra, Sunil, K. Misra. 2011. Equilibrium studies on bivalent transition metal chelates with biologically active schiff's base of heterocyclic aldehydes. Ras. J. Chem. 4, (2), 303-319.

[15] Anil, B. Naik, Maroti, Narwade. 2008. Studies on influence of dielectric constants on complex equilibria between substituted pyrazalines and lanthanide metal ions pH-metrically.Amer.-Euras. scient. Res. 3, (2), 212-216. 